\title{
Texture based Anisotropic Diffusion for Real Ultrasound Image Despeckling
}

\author{
Jie Huang, Xiaoping Yang \\ Department of Mathematics, Nanjing University of Science and Technology, Nanjing, P. R. China
}

\begin{abstract}
This paper presents a new texture based anisotropic diffusion method for real ultrasound image despeckling. Texture information is obtained by a real ultrasound image model. Unlike traditional anisotropic diffusion methods usually taking image gradient as a diffusion index, we take the image texture as a new diffusion index. The results comparing our new method with others on both simulated image and real ultrasound images are reported, and our method shows the superiority in keeping important features of real ultrasound images.
\end{abstract}

Index Terms: Real ultrasound image; anisotropic diffusion; texture; despeckling

(C) 2011 Published by MECS Publisher. Selection and/or peer review under responsibility of the Research Association of Modern Education and Computer Science.

\section{Introduction}

Ultrasound imaging is a non-invasive modality for imaging organs and soft tissue structures in the human body. However, medical ultrasound images are inherently affected by multiplicative speckle noise, which damages resolution and affects the tasks of human interpretation and computer-aided scene analysis. As a result, speckle reduction has become an important issue in medical ultrasound image processing and application.Ease of Use

Various spatial-domain filters have been proposed for the speckle reduction[1][2]. However, the performance of these filters is heavily dependent on the choice of the size and orientation of the local window. And then, statistical based methods[3], variational methods[4][5], PDE based methods[6] and wavelet based methods[7][8] are introduced to deal with speckle noise. Although above methods can achieve good speckle reduction performance, they generally oversmooth ultrasound images and exhibit some limitations in feature preservation, such as image texture, which is very important in clinical diagnosis. In this paper, we focus on using anisotropic diffusion method for speckle reduction in ultrasound image with texture preservation. We will unify image texture information in a diffusion equation. Unlike Pernona and Malik[10] model which

* Corresponding author.

E-mail address: colourfulhj@163.com, yangxp@mail.njust.edu.cn 
depends on magnitude of image gradient, our method is texture sensitive. The experiments show that the capability of our method in keeping image feature.

\section{Texture based anisotropic diffusion}

A. Ultrasound Image Texture

In [9], the authors proposed a real ultrasound image model as:

$f=u+\sqrt{u} n$

Where $f$ is the noisy signal, $u$ is the true signal, $n$ is a zero mean, white Gaussian noise with variance $\delta_{n}^{2}$. Here, we consider $u$ as the product of a constant $u_{c}$ and its texture part $R$, that is $u=u_{c} R$. Where $u_{c}$ can be seen as the mean of the signal, and it's natural to assume that the mean of texture is equal to one. Thus, (1) can be rewritten as:

$f=u_{c} R+\sqrt{u_{c} R} n$

Then, we have:

$\delta_{f}^{2}=E\left(f^{2}\right)-E^{2}(f)$

$$
=E\left(u_{c}^{2} R^{2}+2 u_{c} R \sqrt{u_{c} R} n+u_{c} R n^{2}\right)-E^{2}\left(u_{c} R+\sqrt{u_{c} R} n\right)
$$

Considering $R$ and $n$ are independent,

$$
\begin{aligned}
\delta_{f}^{2} & =u_{c}^{2} E\left(R^{2}\right)+2 u_{c}^{\frac{3}{2}} E\left(R^{\frac{3}{2}}\right) E(n)+u_{c} E(R) E\left(n^{2}\right) \\
& -u_{c}^{2} E^{2}(R)-u_{c} E^{2}(\sqrt{R}) E^{2}(n) \\
& =u_{c}^{2} E\left(R^{2}\right)+u_{c} E\left(n^{2}\right)-u_{c}^{2} E^{2}(R) \\
& =u_{c}^{2} \delta_{R}^{2}+u_{c} \delta_{n}^{2}
\end{aligned}
$$

Where $\delta_{R}^{2}$ is the variance of the texture. And, we have:

$\delta_{R}^{2}=\frac{\delta_{f}^{2}-u_{c} \delta_{n}^{2}}{u_{c}^{2}}$

In a homogeneous region, $u$ is equal to $u_{c}$ and $\delta_{R}^{2}$ is equal to zero; more inhomogeneous the region is, bigger $\delta_{R}^{2}$ is(see Fig.1). And, inhomogeneous regions usually reflect important clinical diagnosis information, such as edges, lesions, tumors and so on. Therefore, we should keep signal information in regions with larger $\delta_{R}^{2}$ while despeckling. 


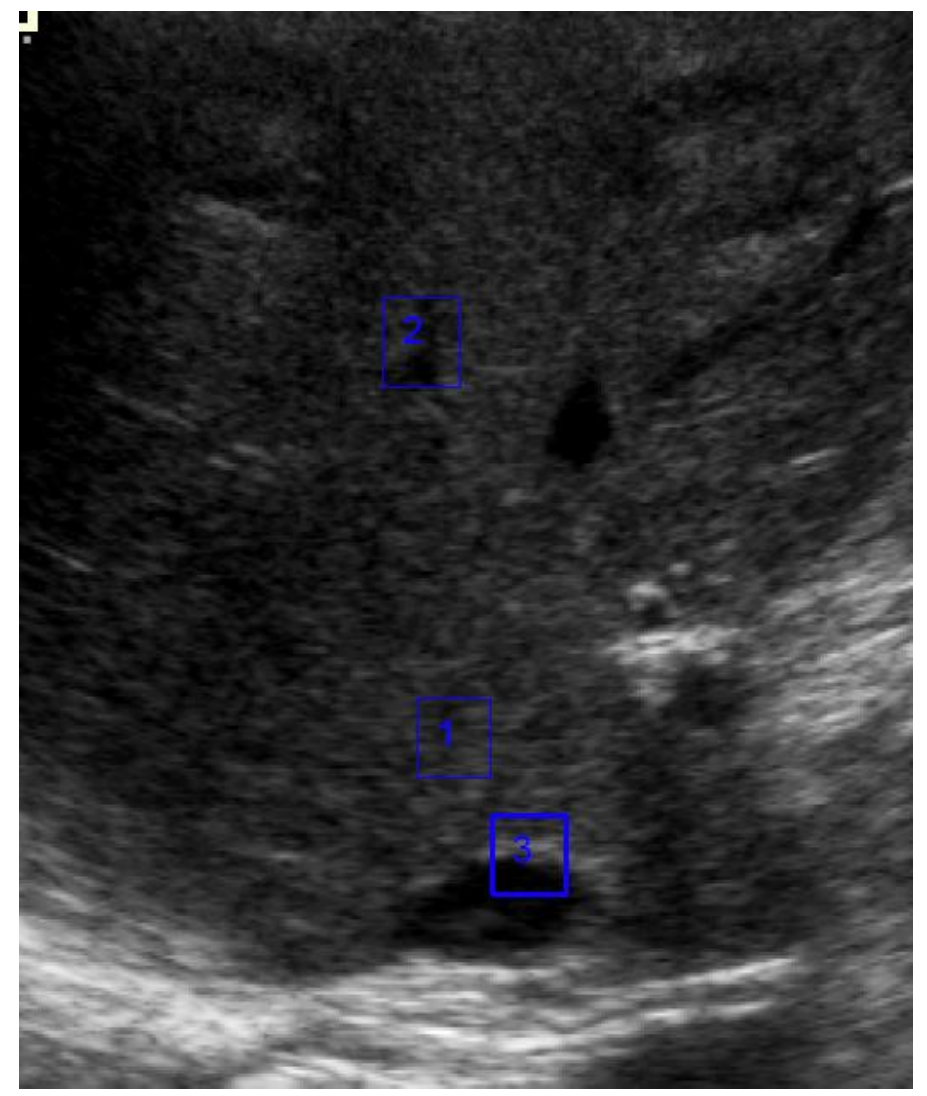

Fig.1 Selected different regions of a real ultrasound image. Region1:homogeneous region,

$\delta_{1}^{2}=134.1$ is caused only by noise; Region 2 : region with small texture,

$\delta_{1}^{2}=180.7$ is the co-effect of noise and texture; Region3: regions with edges,

$\delta_{1}^{2}=578.6$, edge is large texture.

\section{B. Texture based Anisotropic Diffusion}

Perona and Malik [10] proposed the following nonlinear PDE for smoothing image on a continuous domain(PM model):

$$
\left\{\begin{array}{l}
\frac{\partial u}{\partial t}=\operatorname{div}[c(|\nabla u|) \nabla u] \\
u(t=0)=f
\end{array}\right.
$$

where $\nabla$ is the gradient operator, div the divergence operator, $\|$ denotes the magnitude, $c(x)$ the diffusion coefficient, and $f$ the initial image. Where $c(x)$ is suggested to selected as:

$$
c(x)=\frac{1}{1+(x / K)^{2}}
$$

or 


$$
c(x)=e^{-(x / K)^{2}}
$$

Where $K$ serves as an edge magnitude parameter. PM model performs well for images corrupted with additive noise. However, the use of gradient magnitude as an edge detector is not applicable in ultrasound images which is corrupted by speckle noise. In [6], the authors proposed a speckle reducing anisotropic diffusion(SRAD) method by replacing the gradient magnitude with an estimation of the standard deviation of noise. SRAD method is less dependence on the norm of the gradient and reduce the diffusion as the estimated standard deviation of noise decreasing. Unlike SRAD method using noise deviation as feature information, in this paper, we will take use of image texture information. Following (5), the texture at pixel $(i, j)$ of image $u$ is defined as:

$$
\delta_{R(i, j)}^{2}=\frac{\delta_{u_{W(i, j)}}^{2}-u_{W(i, j) c} \delta_{n}^{2}}{u_{W(i, j) c}^{2}}
$$

where $W(i, j)$ represents a window centered at $(i, j), \delta_{u_{W(i, j)}}^{2}$ is the variance of $u$ in window $W(i, j)$, and $u_{W(i, j) c}$ is the mean of $\mathrm{u}$ in window $W(i, j)$. Approximately, we have

$$
u_{W(i, j) c} \approx\left(G_{\sigma} * u\right)_{(i, j)}
$$

and $\delta_{u_{W(i, j)}}^{2}=u_{W(i, j) c}^{2}-\left(u_{W(i, j) c}\right)^{2} \approx\left(G_{\sigma} * u^{2}\right)_{(i, j)}-\left(G_{\sigma} * u\right)_{(i, j)}^{2}$ Where $G_{\sigma}$ is the Gauss kernel with variance $\sigma^{2}$. Thus, we consider following texture based anisotropic diffusion model:

$$
\left\{\begin{array}{l}
\frac{\partial u}{\partial t}=\operatorname{div}\left[c\left(\left|\delta_{R}^{2}\right|\right) \nabla u\right] \\
u(t=0)=f
\end{array}\right.
$$

where

$c\left(\delta_{R}^{2}\right)=\frac{1}{1+\left(G_{\sigma} * u^{2}-\left(G_{\sigma} * u\right)^{2}-\delta_{n}^{2} G_{\sigma} * u\right) /\left(K G_{\sigma} * u\right)^{2}}$ is the diffusion coefficient based on image texture information and $\delta_{n}^{2}$ can be estimated at each iteration using the method introduced in[6].

\section{Numerical Method}

For a two dimensional image $u(i, j)$; with $1 \leq i \leq M ; 1 \leq j \leq N$. Let

$$
\nabla u_{i, j}=\left(u_{i, j}^{x}, u_{i, j}^{y}\right) \text {, }
$$

where

$$
\begin{aligned}
& u_{i, j}^{x}=\left\{\begin{array}{l}
u_{i+1, j}-u_{i, j}, i<M \\
0, i=M
\end{array}\right. \\
& u_{i, j}^{y}=\left\{\begin{array}{l}
u_{i, j+1}-u_{i, j}, j<N \\
0, j=N
\end{array}\right.
\end{aligned}
$$

And we introduce a discrete divergence defined as in[11], 


$$
\begin{aligned}
\operatorname{div}\left(p^{1}, p^{2}\right)_{i, j} & =\left\{\begin{array}{l}
p_{i, j}^{1}-p_{i-1, j}^{1}, 1<i<M \\
p_{i, j}^{1}, i=1 \\
-p_{i-1, j}^{1}, i=M
\end{array}\right. \\
& +\left\{\begin{array}{l}
p_{i, j}^{2}-p_{i, j-1}^{1}, 1<j<N \\
p_{i, j}^{2}, j=1 \\
-p_{i, j-1}^{2}, j=N
\end{array}\right.
\end{aligned}
$$

Then, the numerical approximation to $(8)$ of pixel $(i, j)$ is:

$$
u_{i, j}^{n+1}=u_{i, j}^{n}+\Delta t\left[\operatorname{div}\left(c_{i, j}^{n}\left(u^{n}\right)_{i, j}^{x}, c_{i, j}^{n}\left(u^{n}\right)_{i, j}^{y}\right)\right]
$$

\section{Experiment Results}

In this part, we will compare our despeckling result with other two diffusion methods, PM model and SRAD method. The despeckling performance and texture preserving ability are evaluated with edges detected by canny edge detector.

Fig.2 shows the experiments on a simulated noisy image. Top left is a simulated original image, while top middle is the noisy version of original image simulated by (1). Top right is the corresponding edges of noisy image detected by canny edge detector, and it has many false edges which caused by speckle noise. Middle row(from left to right) are despecking result of PM model, SRAD method and our texture based method respectively, and bottom row(from left to right) are corresponding canny edges of denoised images. PM model, SRAD method and our method can remove speckle effectively. However, PM method destroys edges of the small black circle. The result of SRAD method is close to our result except a few of false edges in second white circle.

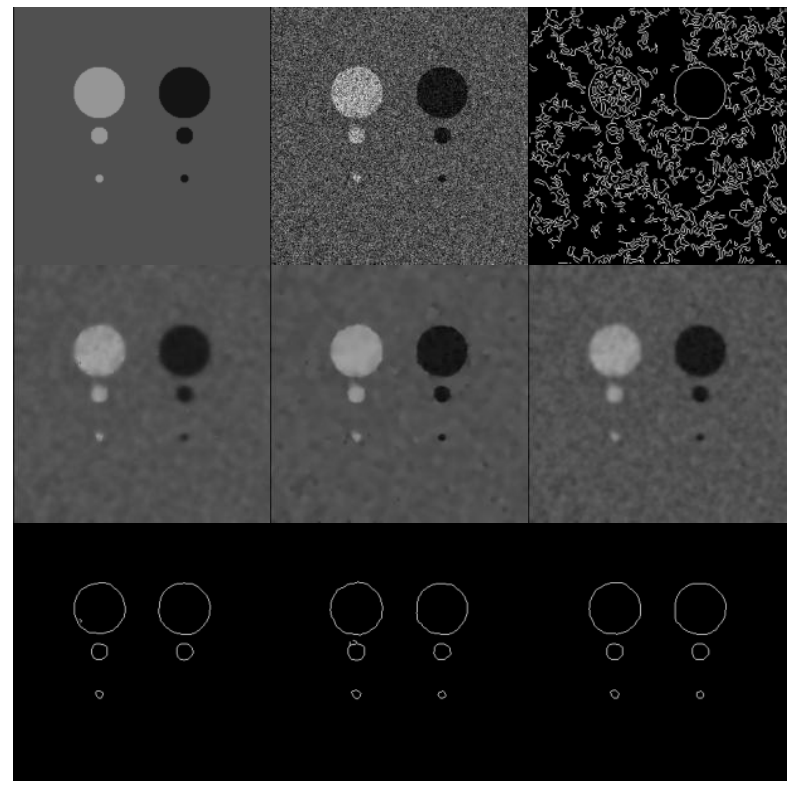

Fig.2 Despeckling example of a simulated image. 
Top Left: simulated original image; Top middle: simulated noisy image using (1);

Top right: corresponding edges of noisy image using canny edge detector;

Middle(from left to right): Despeckling results using PM model, SRAD method and our method respectively;

Bottom: detected canny edges of corresponding denoised images.

Fig.3, Fig.4 and Fig.5 are despeckling results implemented on real ultrasound images. Top left is the real ultrasound image. Top middle are selected regions(blue rectangles) including important features in ultrasound images, which are expected to be reserved in despeckling process. Top right are edges of real ultrasound image detected by canny edge detector. Middle row(from left to right) are despecking results of PM model, SRAD method and our texture based method respectively, and bottom row(from left to right) are corresponding canny edges of denoised images. We can see that our method is superior in protecting image features in selected regions than the other two methods in despeckling process.

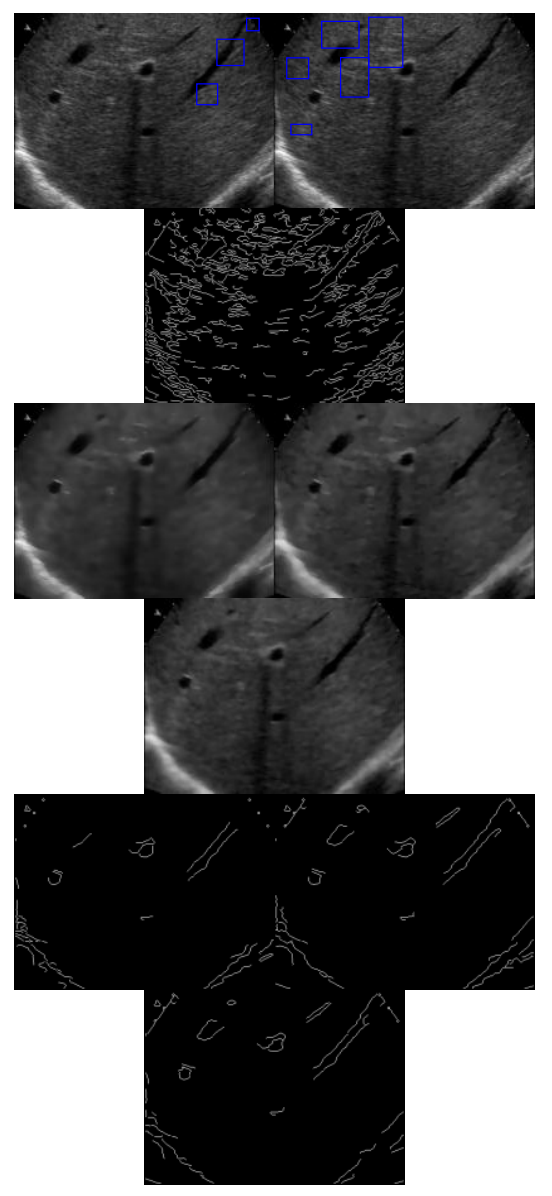

Fig.3 Despeckling of a real ultrasound image

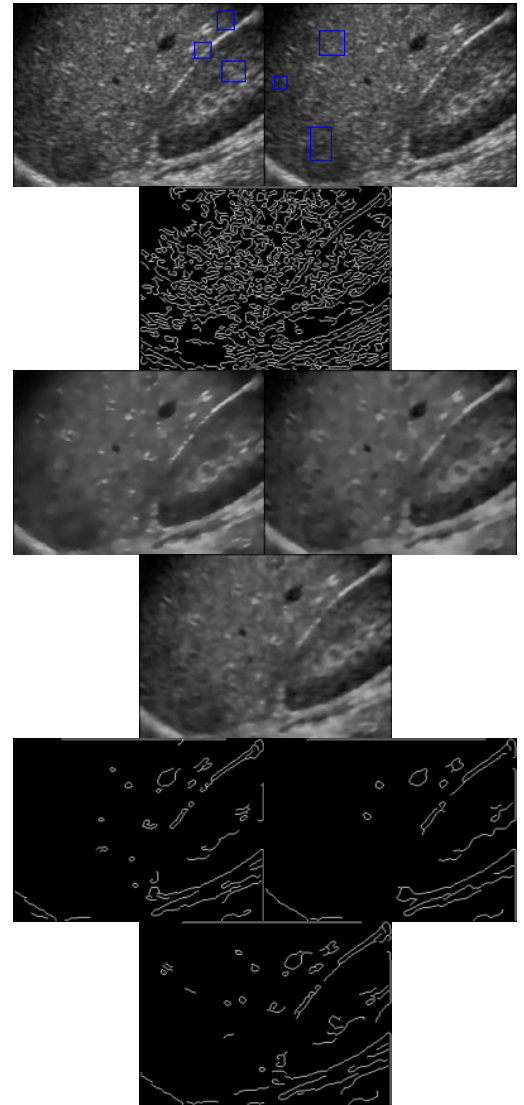

Fig.4 Despeckling of a real ultrasound image 


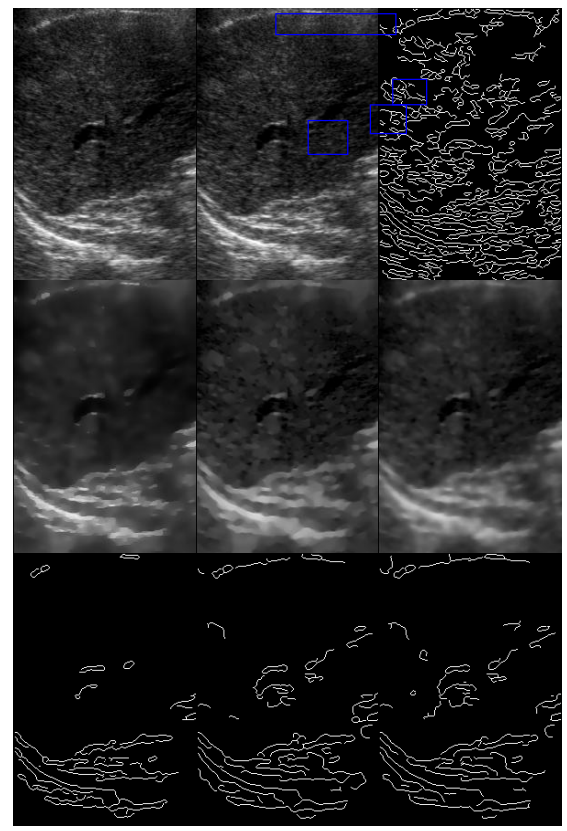

Fig.5 Despeckling of a real ultrasound image

\section{Conclusion}

This paper presents a texture based anisotropic diffusion method for real ultrasound image despeckling. The texture of ultrasound image is obtained from (1). The despeckling performance and feature preserving ability of our method is compared with PM model, and SRAD method. The experiments show the superiority of our method in speckle reduction and feature protection.

\section{Acknowledgment}

The authors would like to thank PLA Nanjing General Hospital for providing ultrasound images.

\section{References}

[1] J. S. Lee, "Digital image enhancement and noise filtering by use of local Statistics", IEEE Trans. Pattern Anal. Mach. Intell., vol. PAMI-2, no. 2, pp. 165-168, Mar. 1980.

[2] V. S. Frost, J. A. Stiles, K. S. Sanmugan, and J. C. Holtzman, "A model for radar images and its application to adaptive digital filtering ofmultiplicative noise", IEEE Trans. Pattern Anal. Mach. Intell., vol. PAMI-4, no. 2, pp. 157-166, Mar. 1982.

[3] Tuncer C. Aysal and K. E. Barner, "Rayleigh-Maximum-Likelihood filtering for speckle reduction of ultrasound images", IEEE Trans. Med. Imag., vol. 26, no. 5, pp. 712-727, Mar. 2007.

[4] L. Rudin, P. L. Lions and S. Osher, "Multiplicative Denoising and Deblurring:Theory and Algorithms", in: S.Osher, N.Paragios(Eds.), Geometric Level Sets in Imaging, Vision and Graphics, Springer, pp. 103119, 2003.

[5] G. Aubert, J. F. Aujol, "A variational approach to removing multiplicative noise", SIAM J. Appl. Math., vol. 68, pp.925-946, 2008. 
[6] YongjianYu, Scott T.Acton, "Speckle reducing anisotropic Diffusion",IEEE Trans. Image Processing, vol. 11, no. 11, pp.1260-1270, 2002.

[7] Xiao Hao, Shangkai Gao and Xiaorong Gao, "A novel multiscale nonlinear thresholding method for ultrasonic speckle suppressing", IEEE Trans. Med. Imag., vol. 18, no. 9, pp.787-794, Sep. 1999.

[8] A. Achim, A. Bezerianos and P. Tsakalides, "Novel Bayesian multiscale method for speckle removal in medical ultrasound images", $\quad$ IEEE Trans. Med. Imag., vol. 20, no. 8, pp.772-783, Aug. 2001.

[9] T. Loupas, W. N. Mcdicken and P. L. Allan, "An adaptive weightedmedian filter for speckle suppression in medical ultrasonic images", IEEE Trans. Circuit and Systems, vol. 36, no. 1, pp.129-135, Jan. 1989.

[10] P. Perona, J. Malik, "Scale space and edge detection using anisotropic diffusion", IEEE Trans. Pattern Anal. Machine Intell., vol. 12, pp. 629-639, 1990.

[11] [11] Antonin Chambolle, "An algorithm for total variation minimization and applications", J. Math. Imag. Vis., vol. 20, pp. 89-97, 2004. 